\title{
Muti-technique observations and modelling of the gas and dust phases of protoplanetary disks
}

\author{
C. Pinte ${ }^{1,2}$, F. Ménard ${ }^{2}$, G. Duchêne ${ }^{2,3}$ and J. C. Augereau ${ }^{2}$
}

${ }^{1}$ School of Physics, University of Exeter, Stocker Road, Exeter EX4 4QL, United Kingdom ${ }^{2}$ Laboratoire d'Astrophysique de Grenoble, CNRS/UJF UMR 5571, 414 rue de la Piscine, B.P. 53, F-38041 Grenoble Cedex 9, France

${ }^{3}$ Astronomy Dept, UC Berkeley, Berkeley CA 94720-3411, USA

A wide range of high-quality data is becoming available for protoplanetary disks. From these data sets many issues have already been addressed, such as constraining the large scale geometry of disks, finding evidence of dust grain evolution, as well as constraining the kinematics and physico-chemical conditions of the gas phase. Most of these results are based on models that emphasise fitting observations of either the dust component (SEDs or scattered light images or, more recently, interferometric visibilities), or the gas phase (resolved maps in molecular lines). In this contribution, we present a more global approach which aims at interpreting consistently the increasing amount of observational data in the framework of a single model, in order to to better characterize both the dust population and the gas disk properties, as well as their interactions. We present results of such modeling applied to a few disks (e.g. IM Lup, see Figure) with large observational data-sets available (scattered light images, polarisation maps, IR spectroscopy, Xray spectrum, CO maps). These kinds of multi-wavelengths studies will become very powerful in the context of forthcoming instruments such as Herschel and ALMA.

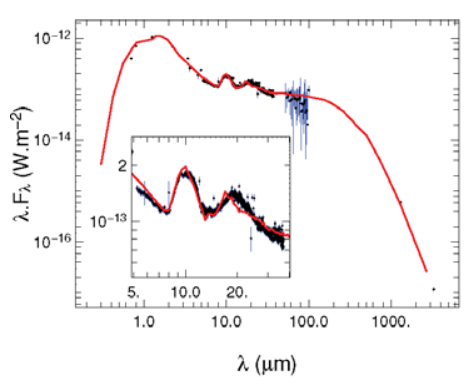

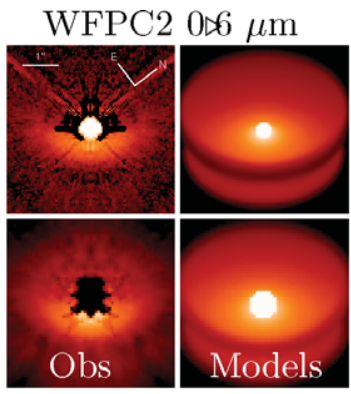

Nicmos $1 \triangleright 6 \mu \mathrm{m}$

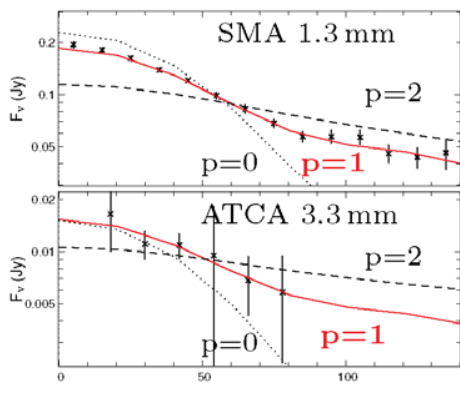

Baseline ( $k \lambda)$

Figure 1. Multi-wavelength modelling of the observations of the disk surrounding IM Lupi. Left panel: SED. The $\mathrm{mm}$ spectral index indicates the presence of large $\mathrm{mm}$-sized grains whereas the silicate bands indicate that micron-sized grains dominate in the disk surface, suggesting a stratified structure for the disk, with small grains at the surface and bigger grains close to the midplane. Central panel: the scattered light images at 0.6 and $1.6 \mu \mathrm{m}$ give strong constraints on the disk geometry. Right panel: millimetre visibilities at 1.3 and $3.3 \mathrm{~mm}$. In the right panel, several models with different surface density $\Sigma(r) \propto r^{-p}$ are presented, only the model with $p=1$ reproduce the observations. The combination of these data allow strong constraints on the model parameters. From Pinte et al 2008, A\&A, 489, 633. 